\title{
Hydroxychloroquine Does not Affect CTLA-4 Expression in LRBA Deficiency: Case Report from Siblings with LRBA Deficiency
}

\author{
Ameera Bukhari', María F. Ortega-Treviño ${ }^{2}$, Nurcicek Padem ${ }^{2}$ and Amer M. Khojah ${ }^{2}$ \\ ${ }^{\prime}$ Department of Microbiology and Immunology, Loyola University Chicago, Chicago, Illinois, USA \\ ${ }^{2}$ Division of Allergy \& Immunology, Ann \& Robert H. Lurie Children's Hospital of Chicago, Chicago, Illinois, USA
}

\begin{abstract}
To the Editor
Lipopolysaccharide-responsive and beige-like anchor protein (LRBA) deficiency is a rare autosomal recessive genetic disease that presents as a primary immunodeficiency and immune dysregulation caused by biallelic mutations in the LRBA gene [1]. LRBA deficiency leads to decreased CTLA-4 (cytotoxic T-lymphocyte-associated protein 4) expression on T regulatory cells (Tregs) due to defective intracellular trafficking of CTLA-4 protein [1,2]. CTLA-4 has been shown to inhibit the immune response by binding to the CD86 (on the antigen-presenting cell) with higher affinity and removing it from the cell surface. The reduction of the CD86 on the antigen-presenting cell leads to impairment of the farther T cell activation [3]. Furthermore, CTLA-4 signaling has been shown to block CD28 (costimulatory signal) mediated $\mathrm{T}$ cell activation and proliferation [4]. CTLA-4 deficient mice die at 3-4 weeks due to uncontrolled T cell proliferation [5].

LRBA deficiency has a broad spectrum of clinical manifestations, such as immunodeficiency from hypogammaglobinemia, lymphoproliferation, and autoimmunity. Lymphoproliferation can present with lymphadenopathy, hepatosplenomegaly, lymphocytic infiltration of non-lymphoid organs such as lung, gut, and brain. Many autoimmune disorders have been reported in LRBA deficiency, such as autoimmune thrombocytopenia, autoimmune hemolytic anemia, arthritis, type 1 diabetes mellitus (DM1), autoimmune thyroid disease, autoimmune enteropathy, atrophic gastritis, hepatitis, vitiligo, psoriasis, myasthenia gravis and uveitis [2,6].

Previous in vitro study has shown a significant increase of CTLA-4 expression on LRBA deficient T cells after overnight culture with chloroquine, an older antimalarial agent [7] However, there is no evidence of such an effect in human subjects after the administration of weight appropriate doses of antimalarial agents. Herein, we presented two siblings with LRBA deficiency and parental consanguinity. CTLA-4 expression was measured before and four weeks after starting hydroxychloroquine.
\end{abstract}

Keywords: Hydroxychloroquine, LRBA Deficiency, CTLA-4, T regulatory cells, autoimmune disorders

\section{Case Report}

Case 1: a 14-year-old East-Indian boy with a history of multiple autoimmune diseases and lymphadenopathy was referred to the Immunology clinic for suspicion of Autoimmune Lymphoproliferative Disease. He was diagnosed with Hashimoto thyroiditis below the age of 2 years and was started on thyroid replacement therapy. His antithyroglobulin antibody level was $>24400 \mathrm{IU} / \mathrm{mL}$ (reference range $<280 \mathrm{IU} / \mathrm{mL}$ ) and his thyroid peroxidase antibodies level was $1883 \mathrm{IU} / \mathrm{mL}$ (reference range $<60 \mathrm{IU} / \mathrm{mL}$ ). At the age of 6 years, he was diagnosed with Diabetes mellitus type 1 and was found to have elevated Glutamic Acid Decarboxylase Antibody $(>30.0 \mathrm{U} / \mathrm{mL}$ Reference range: $<1 \mathrm{U} / \mathrm{mL})$ and Insulin Antibodies $(27 \mathrm{U} /$ $\mathrm{mL}$ Reference range: $<1 \mathrm{U} / \mathrm{mL}$ ). A few years later, he started having intermittent episodes of thrombocytopenia and neutropenia. At the same time, he was found to have cervical and inguinal lymphadenopathy. Because of multiple lymphadenopathies, a lung and neck CT was performed and showed multiple lung nodules and interstitial lung disease in addition to lymphadenopathy within the neck, mediastinum, axillae, retroperitoneum, pelvis and inguinal regions. Primary Immunodeficiency genetic panel, targeted $2^{\text {nd }}$ generation sequencing of 300 immunodeficiency related genes, was done to evaluate for immune dysregulation disorders and revealed a homozygous mutation in LRBA (c.6480-641del). This novel variant resulted in a frameshift and created a premature stop codon 18 amino acids downstream from this location, which may lead to absent or abnormal LRBA protein. The lung biopsy showed interstitial nodular and diffuse lymphoid proliferation. He was started on hydroxychloroquine $6 \mathrm{mg} / \mathrm{kg} / \mathrm{day}$

*Correspondence to: Amer M. Khojah, MD, Division of Pediatric Rheumatology/Allergy and Immunology, Department of Pediatrics, Ann \& Robert H. Lurie Children's Hospital of Chicago, Illinois, USA, 225 East Chicago Avenue, Box 60, Chicago, Illinois 60611, Tel: 773-755-6010, E-mail: akhojah@luriechildrens.org

Citation: Bukhari A, Ortega-Treviño MF, Padem N, Khojah AM (2020) Hydroxychloroquine Does not Affect CTLA-4 Expression in LRBA Deficiency: Case Report from Siblings with LRBA Deficiency. J Pediatr Congenit Dis 6(1): 103. DOI: https://doi.org/10.47275/2379-6707-103.

Received: May 14, 2020; Accepted: June 30, 2020; Published: July 06, 2020

Copyright: (c) 2020 Khojah AM, et al. This is an Open Access article distributed under the terms of the Creative Commons Attribution 4.0 International License (CCBY) (http://creativecommons.org/licenses/by/4.0/) which permits commercial use, including reproduction, adaptation, and distribution of the article provided the original author and source are credited. 
while waiting for insurance approval of abatacept because of the prior in vitro study that shows increase CTLA4 expression with chloroquine and minimal side effect profile. The clinical decision was made not to use steroids because of poorly controlled DM requiring multiple hospitalizations with diabetic ketoacidosis and relatively mild lung disease. He did not have an oxygen requirement, and his adjusted diffusing capacity for carbon monoxide (DLCO) was mildly affected at 79 percentiles. This finding led to the genetic testing of his sister (Case 2) because of her history of multiple autoimmune disorders and the family history of consanguinity.

Case 2: a 13-year-old girl with DM1, autoimmune thyroiditis, lymphadenopathy, psoriasis, psoriatic arthritis, and seizures. Her lung imaging showed pulmonary nodules without interstitial lung disease. The genetic test revealed an identical homozygous mutation of the LRBA gene.

She was started on the same treatment as her brother while waiting for insurance approval of abatacept. CTLA-4 expression on Tregs, soluble interleukin-2 (sIL-2) receptor levels, absolute neutrophil count (ANC), and plasma or serum c-reactive protein (CRP) levels were measured prior to and four weeks after starting hydroxychloroquine treatment. Before treatment, Treg (FOXP3+, CD25hi) accounted for $6 \%$ of CD4 cells in case 1 and $51.8 \%$ of them expressed CTLA-4 (mean fluorescence intensity MFI=333 a.u.) (Figure 1B) which is notably lower than healthy control ( $94 \%$ of Treg express CTLA-4 (Figure 1A). Case 1 had critically low ANC (239 cells/ mm3), elevated CRP $(1.4 \mathrm{mg} / \mathrm{dL}$ - reference range $<0.8 \mathrm{mg} / \mathrm{dL})$ and elevated sIL-2 level $(8510 \mathrm{pg} / \mathrm{mL}$ reference range $<1033 \mathrm{pg} / \mathrm{mL})$. After four weeks of treatment, the percentage of Treg cells and CTLA-4 expression did not change; $5 \%$ and $51 \%$ respectively (MFI of 357 a.u.) (Figure $1 \mathrm{C}$ ) and the ANC was still low at 50 cells $/ \mathrm{mm}^{3}$. On the other hand, soluble IL-2 receptor levels decreased to $2228 \mathrm{pg} / \mathrm{mL}$ (Figure 2) and CRP decreased to normal level $(0.5 \mathrm{mg} / \mathrm{dL}$ ). For Case 2, Treg (FOXP3+, CD25hi) accounted for $4.6 \%$ of CD4 cells before treatment and $3.8 \%$ four weeks after treatment. CTLA4 was expressed on $42 \%$ of these Tregs before treatment (MFI $=298$ a.u.) and increased modestly after treatment to $42.9 \%$ (MFI $=386$ a.u.) (Figure 3). Case 2 had a drop in soluble IL-2 receptor levels from 1265 $\mathrm{pg} / \mathrm{ml}$ to $950 \mathrm{pg} / \mathrm{mL}$ after treatment (Figure 2). Parents provided written consent for publishing the case report.

\section{Discussion}

LRBA deficiency is a rare genetic disease that leads to immune dysregulation due to defective intracellular trafficking of CTLA-4 [7]. The treatment approach of LRBA deficiency consists of bone marrow transplantation, abatacept (CTLA4-Ig fusion protein), immunoglobulin replacement therapy, and other immunosuppressive agents such as sirolimus, cyclosporine, and rituximab [1]. Although bone marrow transplant can be curative, it is associated with significant complications and survival rate of $70.8 \%$ (17 of 24 patients) [8].

To our knowledge, this is the first case report of the effect of hydroxychloroquine as monotherapy on CTLA4 expression in LRBA deficient subjects. The expression of CTLA-4 on T regulatory cells did not change significantly after four weeks of treatment. This finding contradicts the in vitro study, which showed normalization of CTLA-4 expression on T cells after overnight culture with chloroquine [7]. The discrepancy is likely due to the higher concentration of the antimalarial agent in the in vitro culture. The maximum daily dose of hydroxychloroquine in clinical practice is limited due to significant retinal toxicity over long term use. The current recommended dose of hydroxychloroquine seems to be insufficient to change the CTLA-4 expression in a significant way based on the flow cytometry assay. However, soluble IL-2 receptor levels improved dramatically after four weeks of treatment, suggesting that hydroxychloroquine decreases T cell-mediated activation of the immune system by mechanisms other than CTLA-4 expression. Of note, the soluble IL-2 receptor level has been shown to correlate with disease activity in many autoimmune

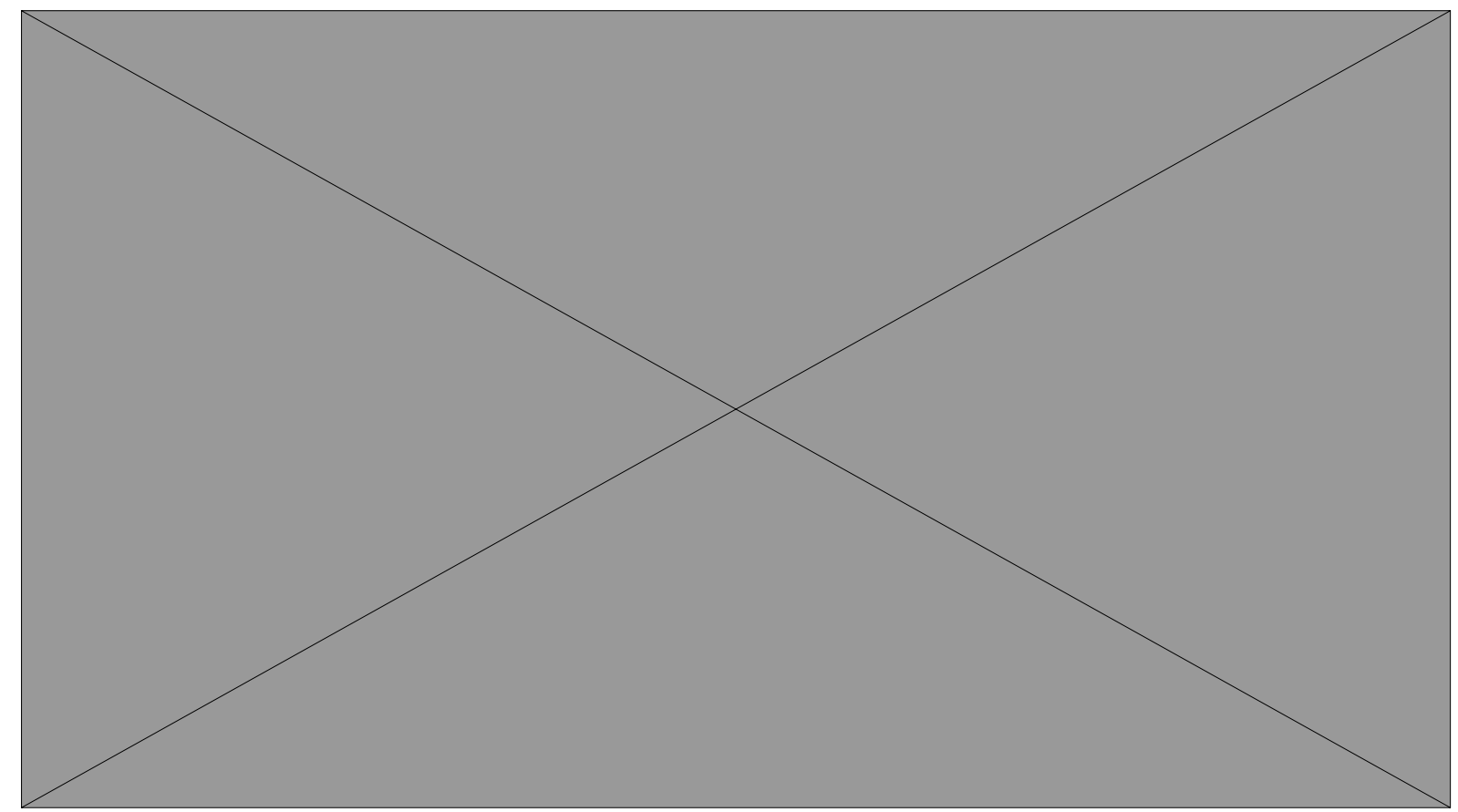

Figure 1: Case 1- CTLA-4 expression on T regulatory (Treg) cells. Panel A shows a healthy control subject with $94 \%$ of T reg expressing CTLA4 protein. Panel B shows only $51 \%$ of T regs in LRBA expressing CTLA4 protein in LRBA deficient subject (Case 1) before treatment. Panel C shows CTLA4 expression on T regs in LRBA deficient subject after four weeks of hydroxychloroquine monotherapy. The CTLA4 did not change significantly with treatment with hydroxychloroquine. 


\section{Case 1}

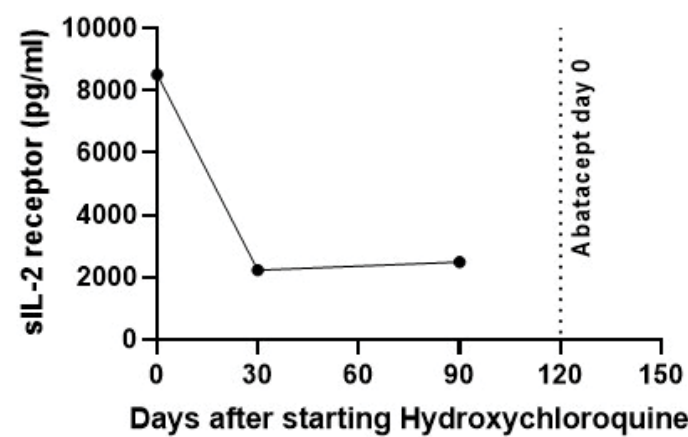

Case 2

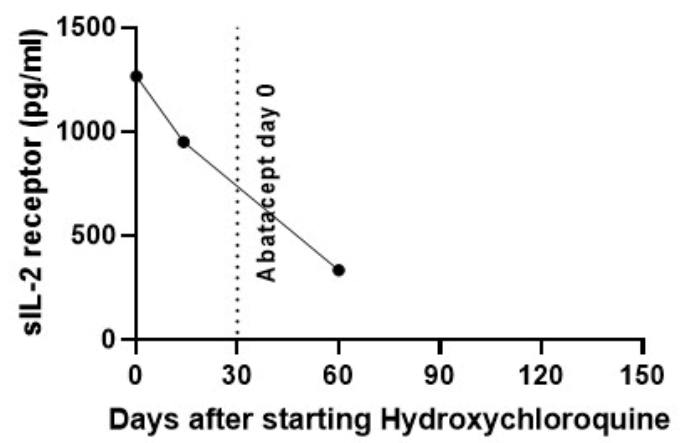

Figure 2: Soluble interleukin-2 (sIL-2) receptor levels in two LRBA deficient subjects. The sIL-2 receptor levels decreased after initiation of hydroxychloroquine monotherapy in both subjects. However, Case 1 sIL-2 receptor did not go back to a normal level $(\leq 1033 \mathrm{pg} / \mathrm{mL})$.

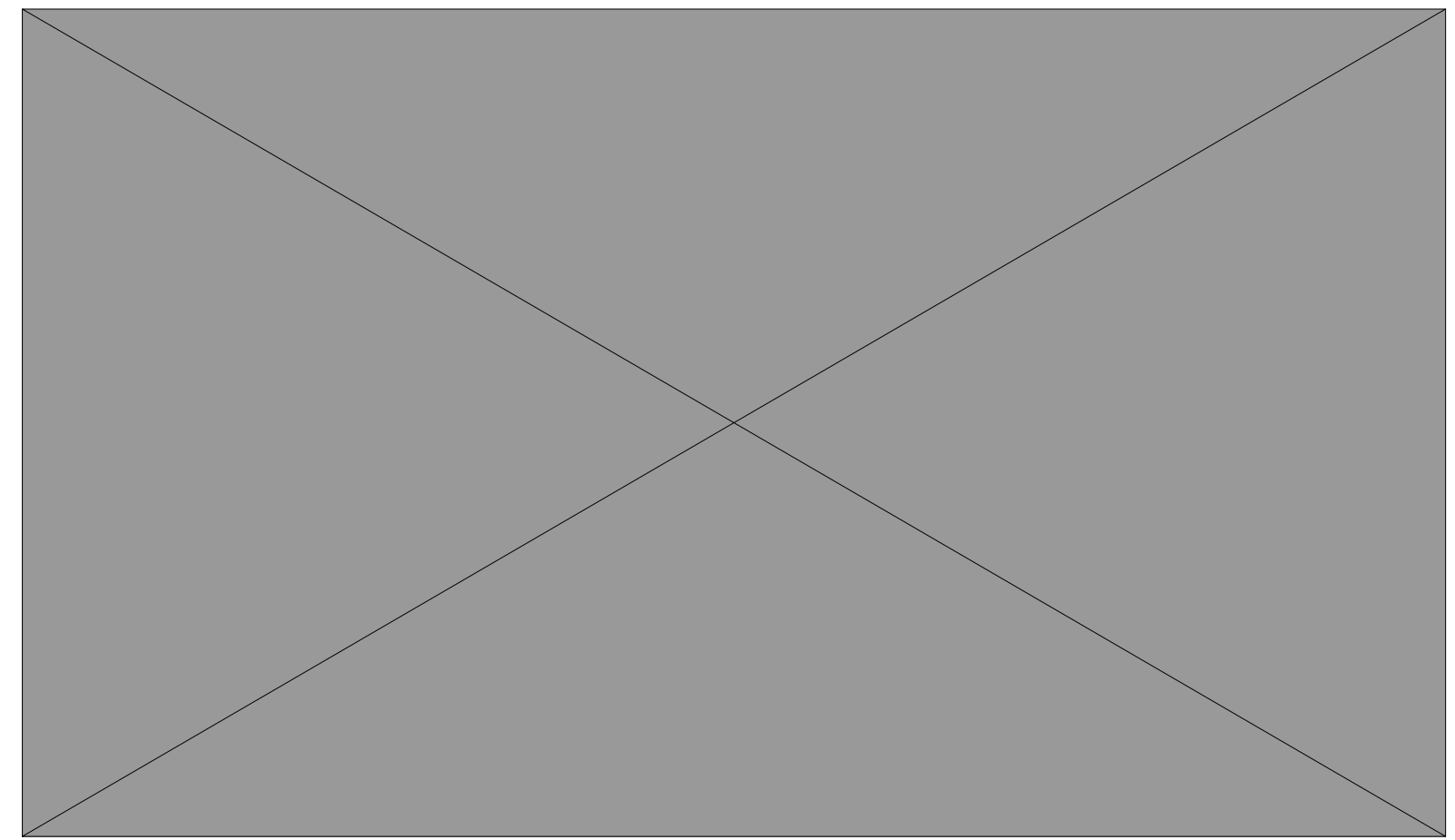

Figure 3: Case 2- CTLA-4 expression on T regulatory (Treg) cells. Panel A shows a healthy control subject with $94 \%$ of T reg expressing CTLA4 protein. Panel B shows only $36 \%$ of T regs in LRBA expressing CTLA4 protein in LRBA deficient subject (Case 2) before treatment. Panel C shows case 2 percentage T regs in LRBA deficient subject after four weeks of hydroxychloroquine. The CTLA4 expression increased slightly (7\%) however it is still below $50 \%$ of the Tregs. 
disorders such as rheumatoid arthritis [9]. However, the utility of this assay in assessing disease activity of LRBA patients is not established. The clinical manifestation of the disease, such as arthritis and neutropenia, did not improve significantly on hydroxychloroquine monotherapy and required the initiation of abatacept. These data indicate hydroxychloroquine can be used as adjunctive therapy but is not enough to control the disease without the addition of other immunosuppressive agents.

\section{Acknowledgement}

We would like to thank Dr. Jhon Routes and his lab member for running the flowcytometry assay to measure the CTLA4 expression.

\section{References}

1. Habibi S, Zaki-Dizaji M, Rafiemanesh H, Lo B, Jamee M, et al. 2019. Clinical, immunologic, and molecular spectrum of patients with LPS-responsive beige-like anchor protein deficiency: asystematic review. J Allergy Clin Immunol Pract 7(7): 2379.e5-2386.e5. https://doi.org/10.1016/j.jaip.2019.04.011

2. Lopez-Herrera G, Tampella G, Pan-Hammarstrom Q, Herholz P, Trujillo-Vargas CM, et al. 2012. Deleterious mutations in LRBA are associated with a syndrome of immune deficiency and autoimmunity. Am J Hum Genet 90 (6): 986-1001. https://doi.org/10.1016/j.ajhg.2012.04.015

3. Qureshi OS, Zheng Y, Nakamura K, Attridge K, Manzotti C, et al. 2011. Trans-endocytosis of CD80 and CD86: a molecular basis for the cell-extrinsic function of CTLA-4. Science 332(6029):600-603. https://doi.org/10.1126/science.1202947

4. Walunas TL, Bakker CY, Bluestone JA. 1996. CTLA-4 ligation blocks CD28-dependent T cell activation. J Exp Med 183(6): 2541-2550. https://doi.org/10.1084/jem.183.6.2541

5. Waterhouse P, Penninger JM, Timms E, Wakeham A, Shahinian A, et al. 1995. Lymphoproliferative disorders with early lethality in mice deficient in Ctla-4. Science 270(5238): 985-988. https://doi.org/10.1126/science.270.5238.985

6. Lo B, Fritz JM, Su HC, Uzel G, Jordan MB, et al. 2016. CHAI and LATAIE: new genetic diseases of CTLA-4 checkpoint insufficiency. Blood 128(8): 1037-1042. https://doi. org/10.1182/blood-2016-04-712612

7. Lo B, Zhang K, Lu W, Zheng L, Zhang Q, et al. Autoimmune disease. Patients with LRBA deficiency show CTLA4 loss and immune dysregulation responsive to abatacept therapy. Science 349(6246): 436-440. https://doi.org/10.1126/science.aaa1663

8. Tesch VK, Abolhassani H, Shadur B, Zobel J, Mareika Y, et al. 2020. Long-term outcome of LRBA deficiency in 76 patients after various treatment modalities as evaluated by the immune deficiency and dysregulation activity (IDDA) score. J Allergy Clin Immunol 145(5): 1452-1463. https://doi.org/10.1016/j.jaci.2019.12.896

9. Caruso C, Candore G, Cigna D, Colucci AT, Modica MA. 1993. Biological significance of soluble IL-2 receptor. Mediators Inflamm 2(1): 3-21. https://doi.org/10.1155/s0962935193000018 